\title{
Long Noncoding RNA GAS5 Acts As A Tumor Suppressor In Laryngeal Squamous Cell Carcinoma Via miR-2I
}

This article was published in the following Dove Press journal: Cancer Management and Research

\author{
Kexing Lyu',* \\ Yang $X u^{1, *}$ \\ Huijun Yue' \\ Yun $\mathrm{Li}^{1}$ \\ Jing Zhao ${ }^{2}$ \\ Lin Chen' \\ Jianhui $\mathrm{Wu}^{3}$ \\ Xiaolin Zhu' \\ Liping Chai' \\ Chunwei $\mathrm{Li}^{1}$ \\ Weiping Wen' \\ Wenbin Lei ${ }^{1}$ \\ 'Department of Otolaryngology, The \\ First Affiliated Hospital of Sun Yat-Sen \\ University, Guangzhou, People's Republic \\ of China; ${ }^{2}$ Department of \\ Otolaryngology, The Third Hospital of \\ Heibei Medical University, Shijiazhuang, \\ People's Republic of China; ${ }^{3}$ Department \\ of Otolaryngology, Meizhou People's \\ Hospital, Meizhou, People's Republic of \\ China
}

*These authors contributed equally to this work
Purpose: Long noncoding RNAs (lncRNAs) have been identified as an important class of noncoding RNAs that are deeply involved in multiple biological processes in tumorigenesis. This study is to investigate the critical roles and biological function of lncRNA growth arrest-specific 5 (GAS5) in tumorigenesis of laryngeal squamous cell carcinoma (LSCC).

Patients and methods: A total of 59 samples of LSCC and paired adjacent tissue, as well as corresponding clinicopathological information were collected. GAS5 expression in both LSCC tissues and human SUN1076 and SNU899 cell lines were analyzed by Real-time quantitative RT-PCR method. Ectopic expression of GAS5 by vector transfection in LSCC cell lines and followed by in vitro experiments was to investigate the critical roles and function of GAS5 in LSCC. Cell Counting Kit 8 (CCK8) assay and PE/7AAD Annexin V Apoptosis analysis was to evaluate cell proliferation ability and cell apoptosis. Co-transfection of GAS5 and miR-21 was to explore the interaction between GAS5 and miR-21 in LSCC. BAX and CDK6 protein level were analyzed by western blot method.

Results: This study demonstrated that GAS5 was significantly downregulated in LSCC tissue and human LSCC cell lines. GAS5 levels were correlated with the clinicopathological features of LSCC patients. In addition, the ectopic expression of GAS5 significantly inhibited cell proliferation and promoted apoptosis. Co-expression analyses indicated that GAS5 is negatively correlated with miR-21 in LSCC tissues. Overexpression of miR-21 eliminated GAS5-mediated cell apoptosis and proliferation suppression. Furthermore, GAS5, which upregulated BAX mRNA expression and downregulated CDK6 mRNA expression, was reversed by ectopic expression of miR-21.

Conclusion: GAS5 suppresses LSCC progression through the negative regulation of miR-21 and its targets involved in cell proliferation and apoptosis, indicating that GAS5 may serve as a biomarker and potential target for LSCC therapy.

Keywords: long noncoding RNA, GAS5, LSCC, miR-21, proliferation, apoptosis

\section{Introduction}

Laryngeal carcinoma is the second most common head and neck cancer and occurs more commonly in men than in women. ${ }^{1}$ With an estimated incidence rate of 5.8/ 100,000 in males, it can seriously threaten health and quality of life. ${ }^{2,3}$ Squamous cell carcinoma is the predominant pathological type, accounting for over $95 \%$ of laryngeal carcinomas. Approximately $60 \%$ of patients present with advanced disease (stage III or IV) once diagnosed, which usually indicates poor outcome and lower treatment efficacy. ${ }^{4}$ Although intervention strategies have greatly improved, the 5-

\footnotetext{
Correspondence: Weiping Wen; Wenbin Lei

Department of Otolaryngology, The First Affiliated Hospital of Sun Yet-Sen University, Yuexiu, Guangzhou,

Guangdong, People's Republic of China

Tel +86208733 3733

Fax +86 2087333108

Email wenwp@mail.sysu.edu.cn;

leiwb@mail.sysu.edu.cn
} 
year survival rate of laryngeal carcinoma has decreased in the past few decades, ${ }^{1}$ indicating that more in-depth investigation is needed to clarify the mechanism of laryngeal squamous cell carcinoma (LSCC) development.

Long noncoding RNA (lncRNA) is a kind of noncoding RNA that usually ranges from $200 \mathrm{nt}$ to over $10 \mathrm{~kb}$ in length. Although lncRNAs were considered "transcriptional noise" in the early years, accumulating evidence indicates that IncRNAs play critical roles in the development of many diseases, especially in tumors. ${ }^{5-7}$ Abnormal expression patterns of $\operatorname{lncRNAs}$ have been indicated to be involved in carcinogenesis. ${ }^{8-10}$ Growth arrest-specific 5 (GAS5) is a nonprotein coding gene that contains multiple C/D box snoRNA genes in its introns. ${ }^{11}$ Mature lncRNA GAS5, an RNA sequence derived from exon 12, regulates the glucocorticoid receptor-associated target gene by competitively binding to the glucocorticoid receptor (GR) and inhibiting glucocorticoid receptor activation. ${ }^{12}$ Studies have revealed that GAS5 acts as a tumor suppressor in multiple biological processes in cancer, including renal cancer, prostate cancer and breast cancer. ${ }^{13-15}$ However, the role and biological function of GAS5 in LSCC remain unknown. miR-21 has been identified as an oncogene in LSCC, which is involved in multiple biological and pathological process in LSCC. ${ }^{16,17}$ However, the underlying mechanism of miR-21 in LSCC need to be furtherly investigated.

In this study, we found that GAS5 is significantly downregulated in LSCC tissue compared with adjacent tissue, which is consistent with the LSCC cell lines. Ectopic expression of GAS5 attenuated proliferation and accelerated apoptosis of LSCC cell lines.

Our further results confirmed that GAS5 is negatively correlated with miR-21 in LSCC tissues. In addition, upregulated GAS5 can negatively regulate miR-21 expression and further regulate miR-21 target genes BAX and CDK6. Then, overexpression of miR-21 can reverse GAS5-mediated proliferation suppression and cell apoptosis. Thus, our study demonstrated that GAS5 acts as a tumor suppressor via negative regulation of miR-21, indicating that GAS5 may be a new target for LSCC therapy.

\section{Materials And Methods}

\section{Clinical Specimens}

A total of 59 samples of LSCC and paired adjacent tissue were obtained from patients in the First Affiliated
Hospital, Sun Yat-sen University. All samples were collected with written informed consent from the patients. The project was approved by the ethics committee of the First Affiliated Hospital of Sun Yat-sen University. All the tissues were collected within 10 min after surgery resection and were immediately transferred to liquid nitrogen. They were stored at $-80^{\circ} \mathrm{C}$ until use. All patients were pathologically confirmed. Clinicopathological information was recorded: age, gender, pathological diagnosis, TNM stage, clinical stage, histological differentiation and distance metastasis.

\section{Cell Culture}

Human LSCC cell lines (SNU899 and SNU1076) were obtained from the Department of Surgery at the University of Hong Kong. Both cell lines were cultured in RPMI-1640 (Gibco) with 10\% fetal bovine serum (FBS, Invitrogen), 100 units $/ \mathrm{mL}$ penicillin, and $100 \mu \mathrm{g} / \mathrm{mL}$ streptomycin in a humidified incubator with $5 \% \mathrm{CO} 2$ at $37^{\circ} \mathrm{C}$.

Both cell lines SUN899 and SNU1076 were approved by the ethics committee of the First Affiliated Hospital of Sun Yat-sen University.

\section{RNA Extraction}

Total RNA was extracted using TRIzol ${ }^{\circledR}$ (Invitrogen, Carlsbad, CA, USA) according to the manufacturer's protocol. RNA purity and concentration were detected by a NanoDrop 2000 spectrophotometer (Thermo Fisher Scientific, Waltham, MA, USA). The purity of the OD260/280 ratio ranged from 1.8 2.0 and was considered acceptable. Reverse transcription was conducted using the Prime Script ${ }^{\mathrm{TM}}$ RT Master Mix Kit (TAKARA BIO INC, Japan) according to the manufacturer's protocol. miRNA reverse transcription proceeded originally from total RNA using the Mir- $\mathrm{X}^{\mathrm{TM}}$ miRNA First-Strand Synthesis Kit (Clontech Laboratories, CA, USA).

\section{Real-Time Quantitative RT-PCR Analysis}

Real-time PCR was performed using a SYBR ${ }^{\circledR}$ Premix Ex Taq $^{\text {TM }}$ (TAKARA BIO INC, Japan) Kit on a LightCycler ${ }^{\circledR}$ 480 (Roche Applied Science, Pennsburg, Germany). The reaction was incubated at $95^{\circ} \mathrm{C}$ for $10 \mathrm{~min}$ followed by 45 cycles of $95^{\circ} \mathrm{C}$ for $15 \mathrm{sec}$ and $60^{\circ} \mathrm{C}$ for $1 \mathrm{~min}$. mRNA expression was normalized to GAPDH. To detect miR-21 expression, U6 was used as an internal control. The primers used in this study are provided in Table S1. Relative

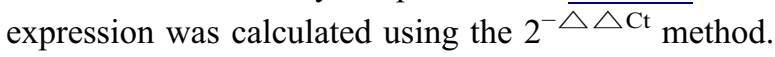




\section{Transfection Assay}

The GAS5 sequence synthesized according to the full length of the GAS5 RNA sequence (NCBI Reference Sequence: NR_002578.3) was cloned into the pHB-EF1MCS-GFP vector (Hanbio Biotechnology, Shanghai, China) to construct the GAS5-pHB plasmid. The empty pHB vector was used as a negative control. The miR-21 mimic was purchased from RiboBio Co., Ltd (Guangzhou, China). Both plasmid and miRNA mimic were transfected into LSCC cell lines using Lipofectamine 2000 reagent (Invitrogen; Thermo Fisher Scientific, USA).

\section{Proliferation Assay}

Pretreated LSCC cells $\left(5 \times 10^{3}\right)$ were seeded into 96-well plates and cultured for 24, 48, 72 and $96 \mathrm{~h}$. For the Cell Counting Kit 8 (CCK8) assay, CCK8 reagent (DOJINDO, Laboratories, Kumamoto, Japan) was added $2 \mathrm{~h}$ before the end of the culture time. Then, the absorbance at $450 \mathrm{~nm}$ was measured using a microplate reader (Bio-Rad, Hercules, CA, USA). Each well was repeated 5 times, and the mean absorbance was used for cell growth curve construction.

\section{Apoptosis Assay}

The apoptosis assay was performed using a PE Annexin V Apoptosis Detection Kit (BD Biosciences, USA). LSCC cell lines transfected with plasmid or miRNA mimic were cultured for $48 \mathrm{~h}$. The cells were harvested by $0.25 \%$ trypsin and washed twice with cold PBS. Then, the cells were stained with annexin v-PE and 7. $\mathrm{AAD}$ and incubated in the dark for $15 \mathrm{~min}$ at $37^{\circ} \mathrm{C}$. Stained cells were finally analyzed by flow cytometry (FACScan; BD Biosciences), and the apoptotic ratio was obtained.

\section{Western Blot Analysis}

The cells were lysed in RIPA buffer (Beyotime Biotechnology, Shanghai, China) on ice for $20 \mathrm{mins}$. Protein concentration was determined using a BCA Detection Kit (Beyotime Biotechnology, Shanghai, China). Proteins were separated on a $12 \%$ sodium dodecyl sulfatepolyacrylamide gel (SDS-PAGE) and then transferred to nitrocellulose membranes (Schleicher and Schuell, Germany). The membranes were incubated in 5\% skim milk for $1 \mathrm{~h}$ at room temperature and washed three times on a shaker in Tris-buffered saline containing 20\%
Tween-20 (TBST). Then, the membranes were incubated with antibodies against BAX (Cell Signaling Technology, Danvers, MA, USA), Rabbit monoclonal anti-CDK6 (cat. no. ab124821; 1:50,000; Abcam, Cambridge, MA, USA), mouse monoclonal anti-GADPH (cat. no. HRP-60004; 1:10,000; Proteintech group, lnc; Manchester, UK) and $\beta$ tubulin rabbit Polyclonal Antibody (cat. no. AF0001; 1:1000; Beyotime Biotechnology, Shanghai, China). Goat anti-mouse IgG-HRP antibody (cat. no. ab A0216; 1:1000; Beyotime Biotechnology, Shanghai, China) and goat antirabbit IgG-HRP antibody (cat. no. ab A0208; 1:1000; Beyotime Biotechnology, Shanghai, China) were used as secondary antibodies. After application of the secondary antibody, the blots were visualized using the electrochemiluminescence (ECL) system (Bio-Rad Laboratories, Hercules, CA, USA).

\section{Statistical Analysis}

All statistical analyses were conducted by SPSS software version 23.0 (SPSS Inc., Chicago, IL, USA). The figures were conducted by GraphPad Prism 6. Student's $t$-test was performed to analyze the differences between the two groups. Chi-square or Fisher's exact tests were used to analyze the association between gene expression and clinicopathological characteristics. $P<0.05$ was considered statistically significant.

\section{Results}

\section{GAS5 Was Significantly Downregulated In LSCC Tissues}

The expression of GAS5 in LSCC was evaluated by qRT-PCR in 59 pairs of LSCC tissues and adjacent normal tissues. As shown in Figure 1A, the GAS5 expression level was significantly lower in LSCC tissues than in adjacent tissues. Further investigation found that GAS5 expression in LSCC cell lines (SNU1076 and SNU899) was consistently lower than in that in normal cell lines (Figure 1B). The clinicopathological features and the expression levels of GAS5 were analyzed. As shown in Table 1, the expression level of GAS5 in cancer tissues was significantly correlated with the $\mathrm{T}$ stage. There was no correlation between GAS5 level and age, gender, lymph node, clinical UICC stage, histological differentiation, smoking and drinking. This initially indicated that low GAS5 expression contributed to LSCC development. 
A

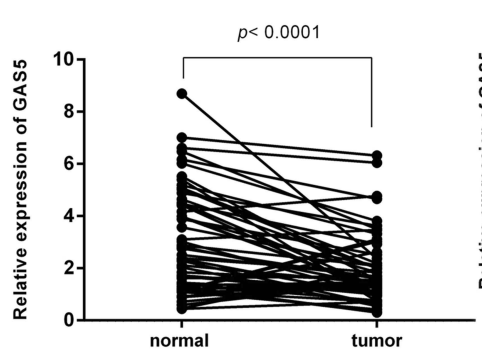

B

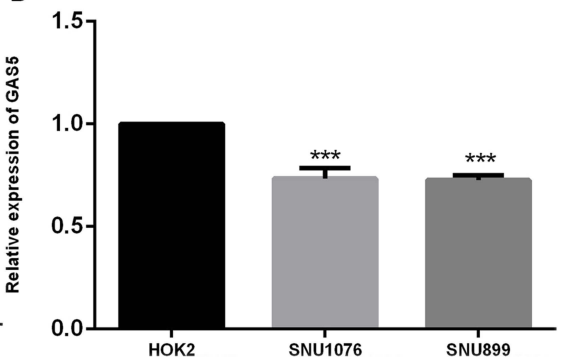

Figure I IncRNA GAS5 expression in LSCC tissues and cell lines. (A) The relative expression of GAS5 in LSCC tissues ( $\mathrm{n}=59$ ) and adjacent normal tissues were measured by RT-qPCR normalized to GADPH. (B) Relative GAS5 expression in LSCC cell lines (SNUI076 and SNU899) and the normal oral epithelial cell line (HOK2) were determined by RT-qPCR. ***p<0.00I.

Table I Relationship Between IncRNA GAS5 Expression And Tumor Clinicopathological Features In LSCC

\begin{tabular}{|c|c|c|c|c|}
\hline Variables & Cases & GAS5 Low Level & GAS5 High Level & P-Valuea \\
\hline \multicolumn{5}{|l|}{ Sex } \\
\hline Male & 58 & 29 & 29 & 1.000 \\
\hline Female & 1 & 1 & 0 & \\
\hline \multicolumn{5}{|l|}{ Age (years) } \\
\hline$<60$ & 28 & 14 & 14 & 0.902 \\
\hline$\geq 60$ & 31 & 16 & 15 & \\
\hline \multicolumn{5}{|l|}{ T stage } \\
\hline TI-3 & 30 & 17 & 25 & $0.012^{\mathrm{a}}$ \\
\hline $\mathrm{T} 4$ & 29 & 13 & 4 & \\
\hline \multicolumn{5}{|l|}{ Lymph node } \\
\hline Positive & 28 & 12 & 16 & 0.401 \\
\hline Negative & 31 & 10 & 21 & \\
\hline \multicolumn{5}{|l|}{ Clinical UICC stage } \\
\hline Early stage (I-II) & 14 & 9 & 5 & 0.249 \\
\hline Advance stage (III-IV) & 45 & 21 & 24 & \\
\hline \multicolumn{5}{|l|}{ Histological differentiation } \\
\hline Well and moderately differentiated & 40 & 19 & 21 & 0.260 \\
\hline Poor and undifferentiated & 19 & 12 & 7 & \\
\hline \multicolumn{5}{|l|}{ Smoking } \\
\hline No & 35 & 18 & 17 & 0.673 \\
\hline Yes & 24 & 11 & 13 & \\
\hline \multicolumn{5}{|l|}{ Drinking } \\
\hline No & 23 & 10 & 13 & 0.486 \\
\hline Yes & 36 & 19 & 17 & \\
\hline
\end{tabular}

Note: ${ }^{\mathrm{a}} \mathrm{P}<0.05$.

\section{GAS5 Acted As A Tumor Suppressor In LSCC Cell Lines}

To investigate the role of GAS5 in LSCC, the LSCC cell lines SNU899 and SNU1076 were transfected with the GAS5-pHB plasmid. qRT-PCR confirmed that the GAS5 levels were largely overexpressed after transfection in both cell lines (Figure 2). CCK8 experiments showed that ectopic expression of GAS5 significantly suppressed the proliferation ability of cell lines (Figure $3 \mathrm{~A}$ and B). In addition, double-stained flow cytometry analysis indicated that overexpression enhanced cell apoptosis compared to negative control group (Figure 4A-D). These 

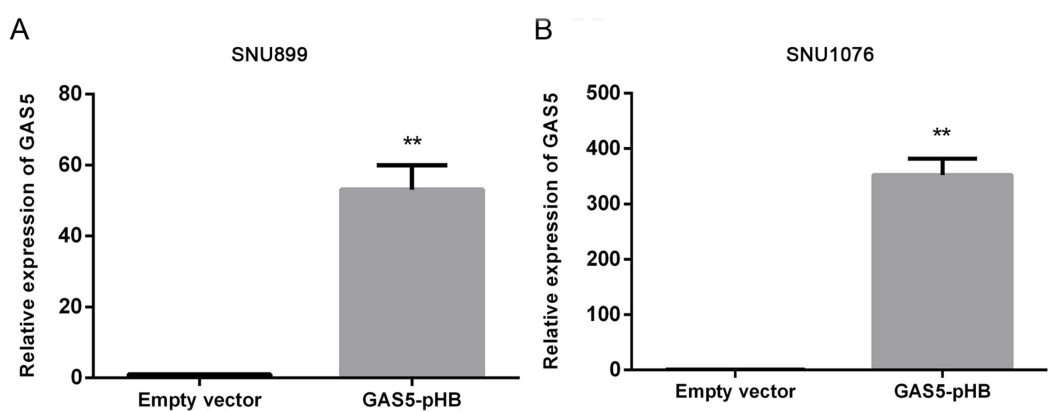

Figure 2 GAS5 expression after cell transfection. (A, B) human cell lines SNU899 and SNUI076 were transfected with empty vector and GAS5-pHB plasmid and then RT-qPCR were performed to confirm the transfection effect. $* * p<0.01$.

A

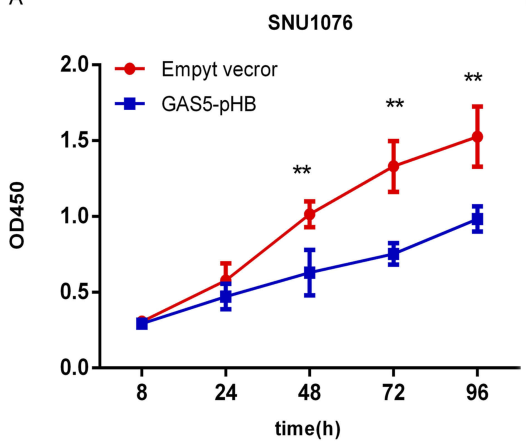

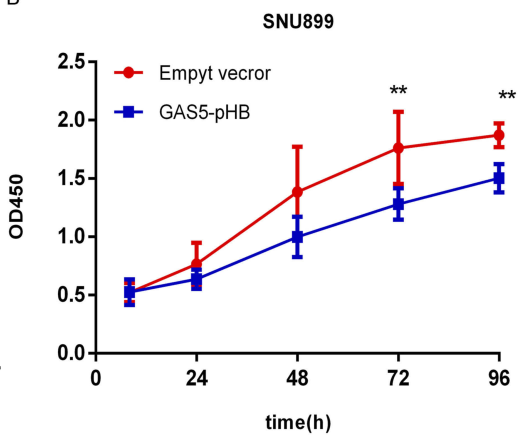

Figure 3 GAS5 suppressed LSCC cell lines proliferation. (A-B) Overexpression of GAS5 decreased cell lines proliferation in both SNUI076 and SNU899 LSCC cell lines. LSCC cell lines were transfected with either empty vector or GAS5-pHB plasmid and then cell lines proliferation ability was measured by CCK 8 assay at $8,24,48,72,96$ hr. $* * \mathrm{p}<0.01$.

results indicated that GAS5 may act as a tumor suppressor in LSCC.

To elucidate the mechanism of GAS5 involvement in proliferation and apoptosis pathways, qRT-PCR results showed that overexpression of GAS5 downregulated bcl 2 and upregulated BAX at the gene expression level (Figure 5A-D). Moreover, upregulation of GAS5 decreased the gene expression of CDK4 and CDK6 in both cell lines (Figure 6A-D). Furthermore, overexpression of GAS5 upregulated BAX and downregulated CDK6 expression by Western blot (Figure 7). To investigate the connection of GAS5 expression and proliferation or apoptosis status in LSCC tissues, we performed correlation analysis between GAS5 and BAX/CDK6. The results indicated that GAS5 was significantly positive correlated with BAX, while negative correlated with CDK6 expression (Figure S1). Collectively, all these results demonstrated that GAS5 regulated BAX and CDK6 expression, thus leading to proliferation suppression and apoptosis enhancement.

\section{GAS5 Suppresses LSCC Cell Lines Through Negative Regulation Of miR-2I}

IncRNAs play important roles in tumor biology via complex mechanisms. ${ }^{18,19}$ Emerging evidence indicates that lncRNAs can regulate biological processes via sponging to miRNAs. ${ }^{5,20}$ miR-21 have been indicated to be involved in carcinogenesis process of LSCC, while the underlying mechanism is not fully understood. Early studies have revealed that GAS5 has a negative connection with miR-21. ${ }^{21}$ Therefore, our further study was carried out to investigate whether GAS5 exerts a suppressible role in LSCC via miR-21.

Through qRT-PCR analysis, miR-21 was found to be significantly upregulated in laryngeal cancer tissues compared to adjacent normal tissues (Figure 8A). Then, co-expression analysis by RT-qPCR indicated that there was negative correlation between GAS5 and miR-21 in LSCC tissues (Figure 8B). Furtherly, miR-21 was confirmed to be directly regulated by GAS5 and upregulated GAS5 significantly downregulated miR-21 


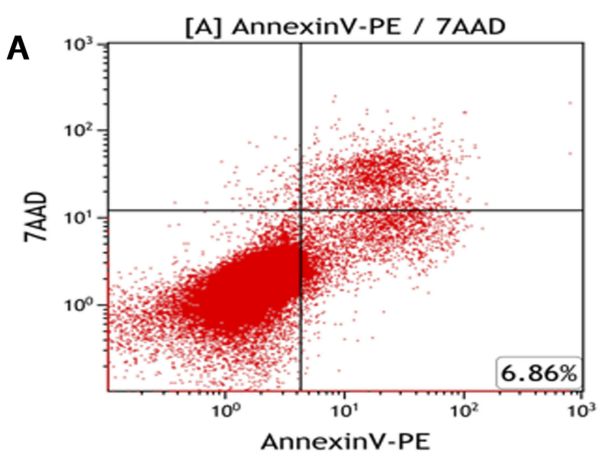

SNU899-Empty vector

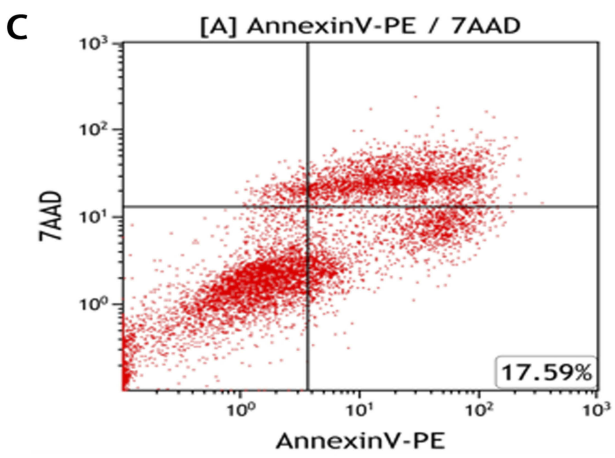

SNU1076-Empty vector

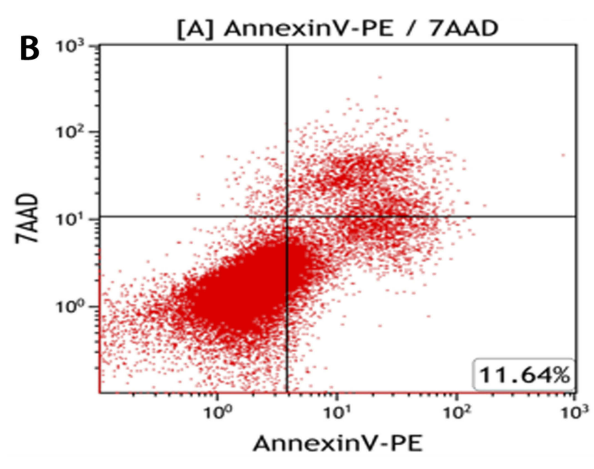

SNU899-GAS5-pHB

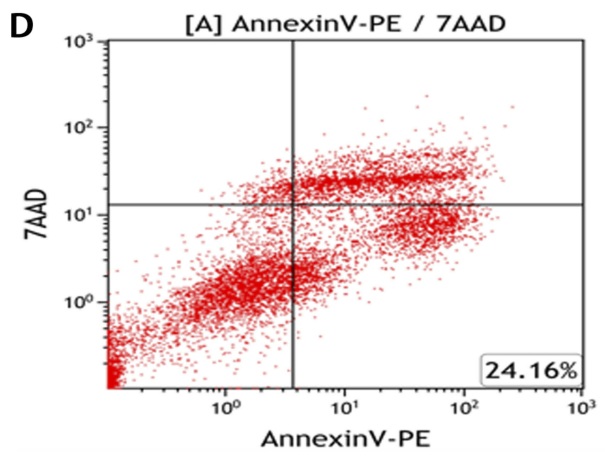

SNU1076-GAS5-pHB

Figure 4 GAS5 promoted LSCC cell lines apoptosis. (A, B) Overexpression of GAS5 increased cell lines apoptosis in LSCC cell lines. At 48 hrs after transfection, LSCC cell lines apoptosis were determined by flow cytometry. Late cell apoptosis rate was significantly increased in cell transfected with GAS5-pHB compared with empty vector in both SNU899 (A, B) and SNUI076 (C, D) cell lines.

expression (Figure 8C and D). To investigate whether GAS5 functionally regulates LSCC via miR-21, we cotransfected GAS5 plasmid and miR-21 mimic into LSCC cell lines. As Figure 9 shows, upregulation of miR-21 reversed the GAS5-media effects, including proliferation suppression and induced apoptosis. Moreover, upregulated miR-21 inhibited BAX expression and increased CDK6 induced by GAS5 overexpression (Figure 7). In conclusion, GAS5 suppresses LSCC through the negative regulation of miR-21, which targets BAX and CDK6, leading to proliferation attenuation and cell apoptosis.

\section{Discussion}

Although the current comprehensive therapy for laryngeal cancer has obviously improved the quality of life of patients, the 5-year survival rate has decreased. ${ }^{2,22}$ Therefore, determining the mechanism of carcinogenesis of laryngeal cancer is very urgent. Accumulating evidence indicates that IncRNAs play important roles in carcinogenesis pathology. For example, aberrant expression of TUG1, NEAT1 and MEG3 plays an important role in neurodegenerative diseases. ${ }^{23} \mathrm{H} 19$ has been demonstrated to have a considerable effect on the pathophysiological process of liver diseases. ${ }^{24}$ The roles of lncRNA in many cancers, including prostate cancer, breast cancer, gastric cancer, liver cancer, and liver cancer glioma, have also attracted much attention. ${ }^{23,25}$ However, little is currently known about the role of lncRNAs in laryngeal cancer. Zhisen Shen investigated the lncRNA expression profiles in laryngeal cancer, but further study is needed to verify these findings. ${ }^{9}$ Another study showed that RP11 serves as a tumor suppressor by regulating $\mathrm{CDH} 1 .^{26}$ The elucidation of the biological function and mechanism of lncRNAs in cancer development is promising for novel cancer therapeutic strategies.

Aberrant expression of IncRNAs contributes to cancer development. Although low expression of GAS5 has been verified to play important roles in variable cancers, such as liver cancer, $^{27}$ breast cancer, ${ }^{13}$ colorectal 


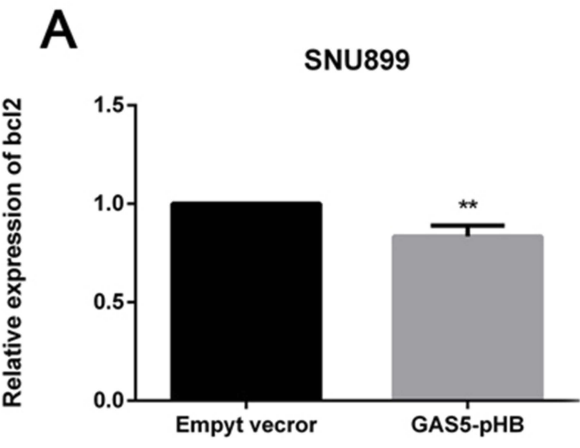

B
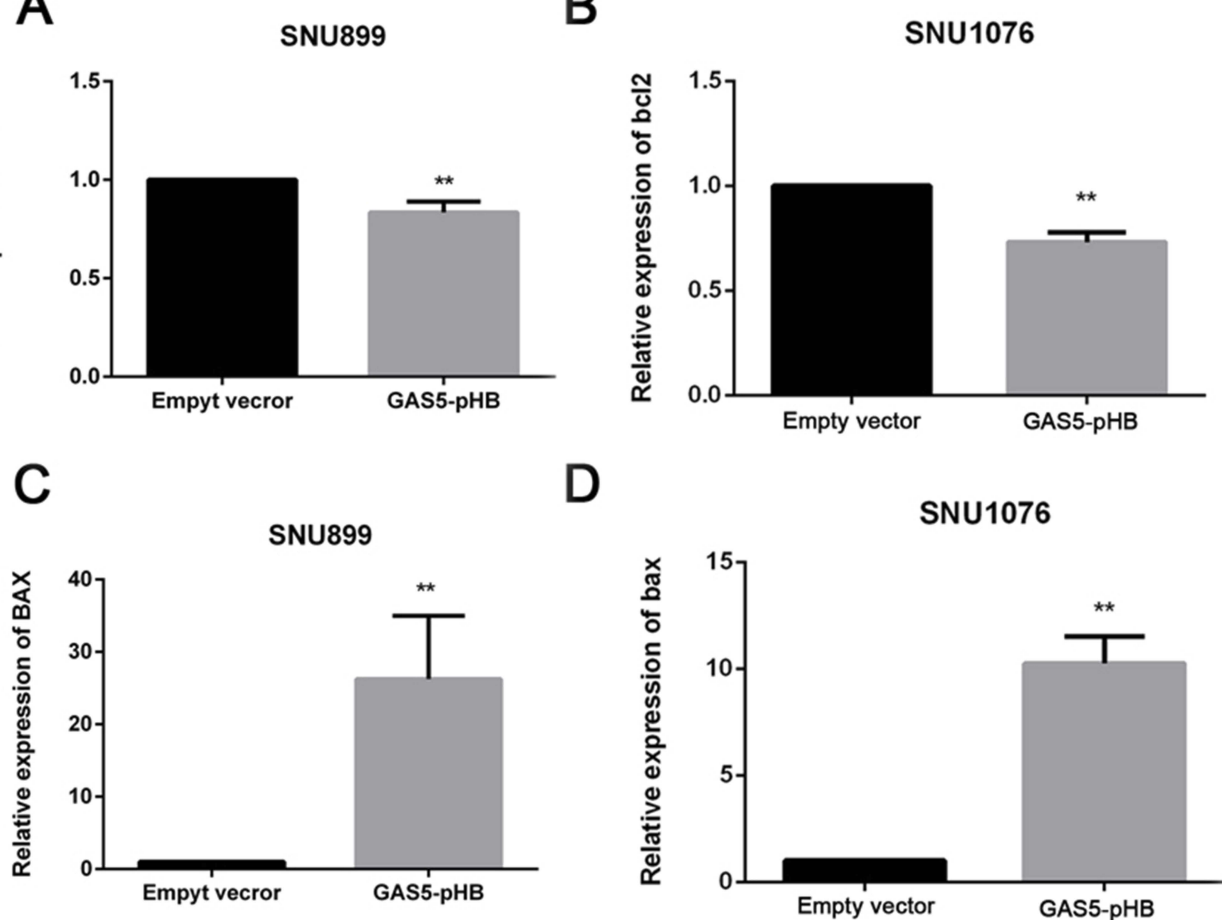

Figure 5 GAS5 suppressed bcl2 and increased BAX gene expression. Bcl2 and BAX gene expression level were detected by RT-qPCR after GAS5 transfection. Bcl2 were decreased in both SNU899 and SNU1076 cell lines (A, B), while BAX were increased (C, D). ${ }^{* *}$ p $<0.01$.

A

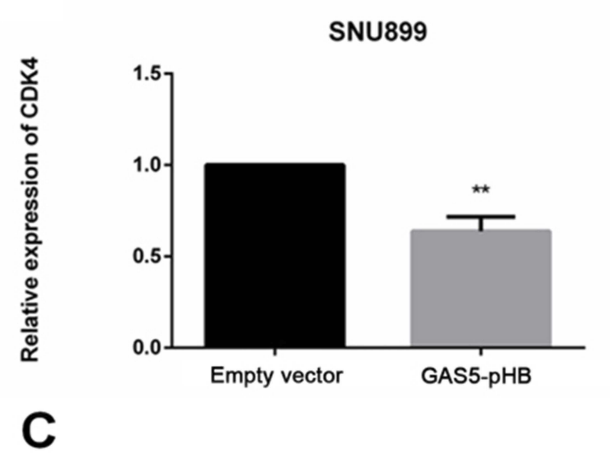

SNU1076

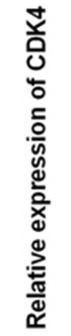

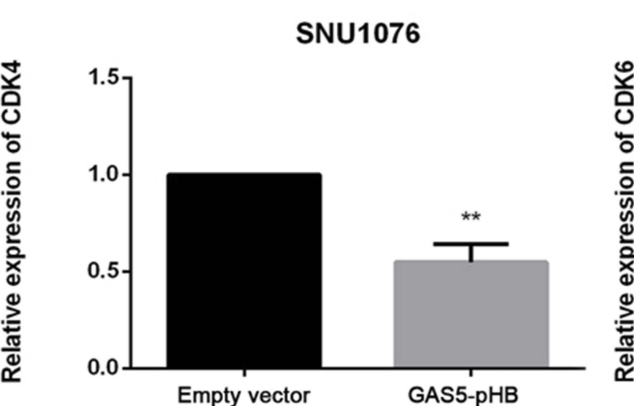

B

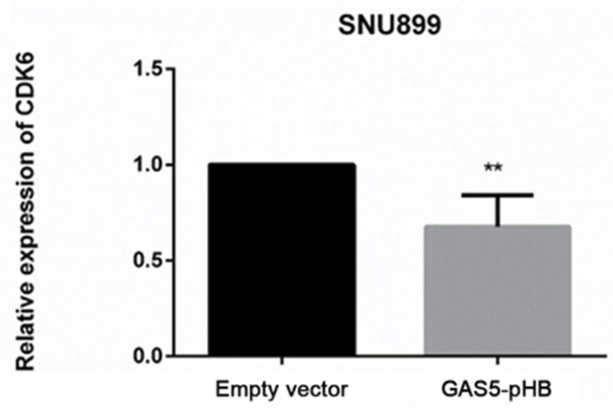

D

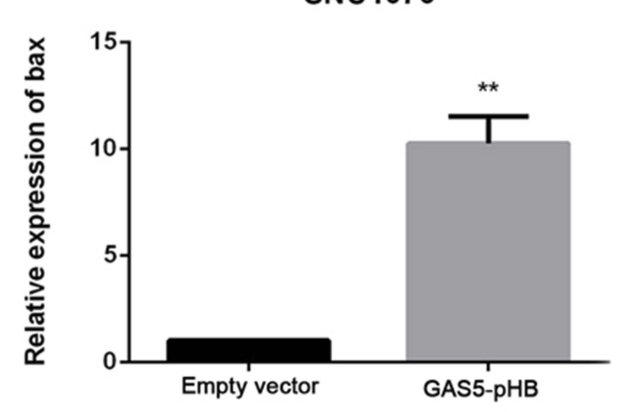

\section{SNU1076}

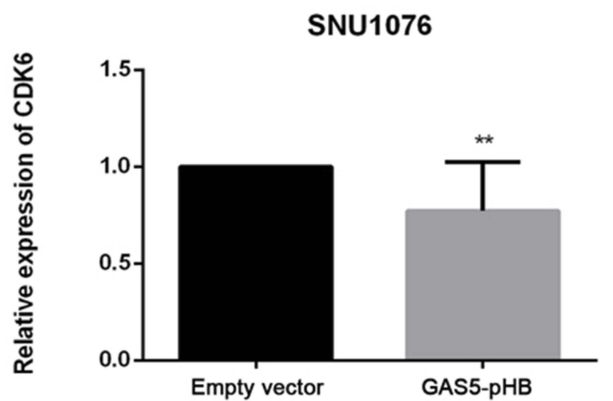

Figure 6 GAS5 decreased CDK4 and CDK6 gene expression. CDK4 and CDK6 gene expression level were detected by RT-qPCR after GAS5 transfection. CDK4 (A, C) and CDK6 (B, D) decreased in both SNU899 and SNU1076 cell lines after GAS5 transfection. **p<0.01. 


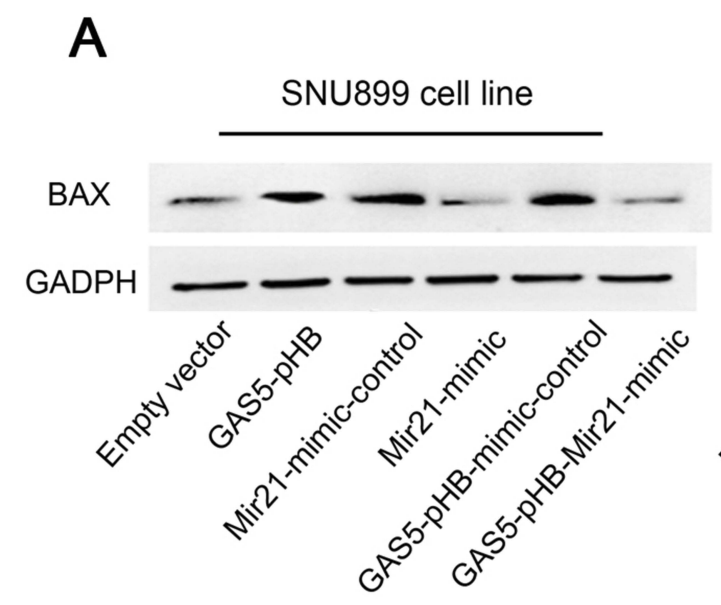

\section{B}
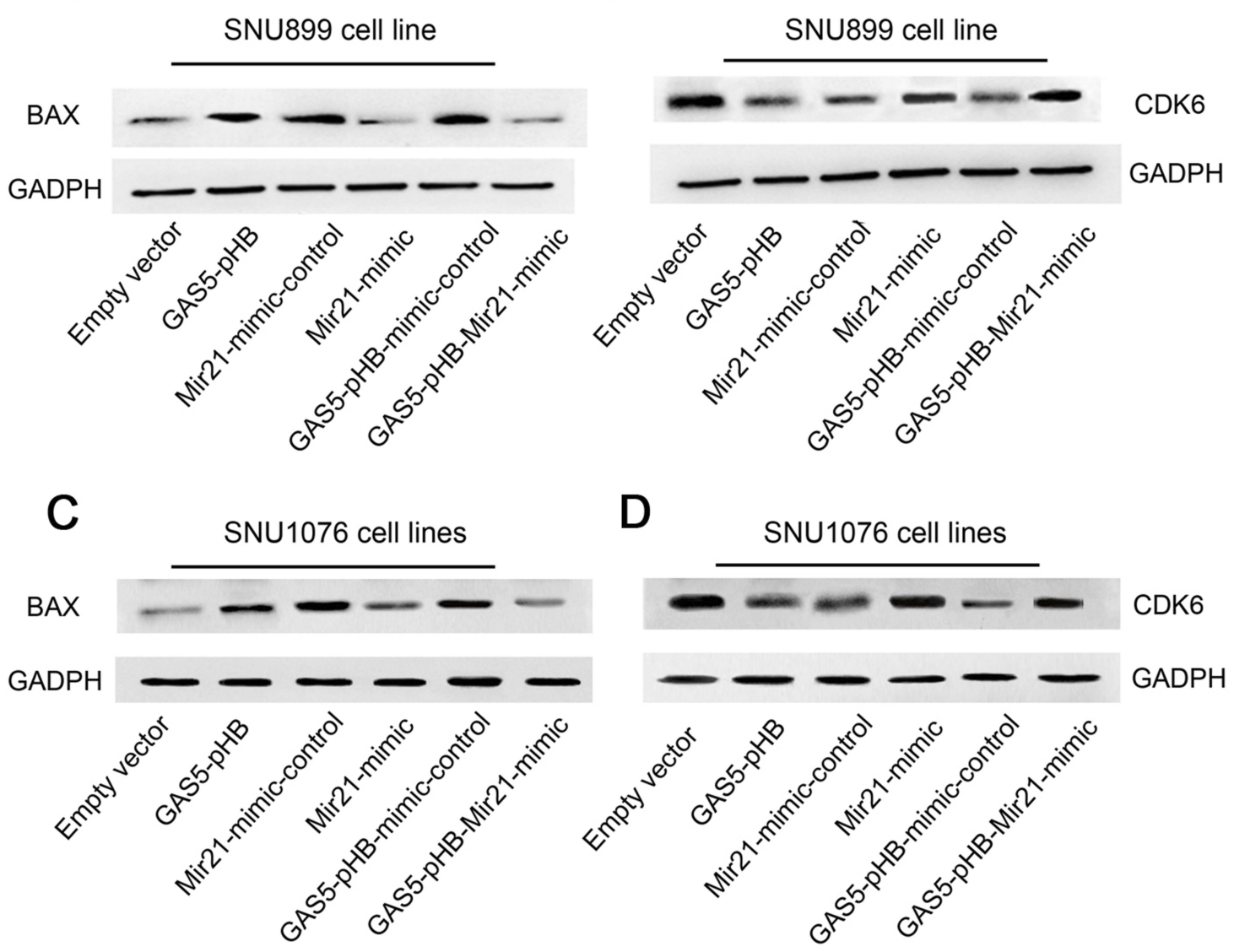

Figure 7 GAS5 regulates cell proliferation and apoptosis via miR-2I. BAX expression levels in SNU899 cells after transfection with empty vector or GAS5 overexpression vector+ miR-21-mimic (A); CDK6 expression levels in SNU899 cells after transfection with empty vector or GAS5 overexpression vector+ miR-2I-mimic (B); BAX expression levels in SNUI076 cells after transfection with empty vector or GAS5 overexpression vector+ miR-2I-mimic (C); CDK6 expression levels in SNUI076 cells after transfection with empty vector or GAS5 overexpression vector+ miR-2I-mimic (D).

cancer $^{28}$ and lung cancer, ${ }^{29}$ the function and role of GAS5 in laryngeal cancer are still unknown. In the present study, we found that GAS5 expression in laryngeal cancer was lower than that in adjacent nontumor tissue. Clinicopathological analysis revealed a correlation between lower expression and late $\mathrm{T}$ stage. This finding indicated that abnormal expression may contribute to the development of laryngeal cancer. Similarly, there was lower GAS5 levels in LSCC cell lines than in nontumor cell lines. Although reports have indicated that GAS5 functions as a tumor suppressor, the functional biological behavior of GAS5 in carcinogenesis varies. GAS5 can regulate apoptosis in prostate cancer and breast cancer, ${ }^{30,31}$ while in lung cancer and gastric cancer, GAS5 regulates proliferation. ${ }^{29,32}$ Another report revealed that GAS5 suppressed invasive ability. ${ }^{27}$ By ectopic expression of GAS5 in LSCC cell lines, we confirmed that GAS5 can suppress cell proliferation and induce cell apoptosis by regulating BAX and CDK6 expression.

lncRNAs can function as endogenous "sponges" by binding to miRNAs and further regulate their target mRNAs. ${ }^{33}$ lncRNA-KRTAP5-AS1 and lncRNA-TUBB2A interact with miR-596 and miR-3620-3p. ${ }^{7}$ DSCR 8 regulates hepatocellular carcinoma via miR-485-5p, ${ }^{34}$ and NEAT1 regulates LIN28B by sponging to miR-506. ${ }^{35}$ Moreover, GAS5 can directly suppress miR-21 expression. ${ }^{21}$ Another study revealed that GAS5 regulated osteoarthritis via miR21. ${ }^{36}$ However, whether the ceRNA mechanism plays a critical role in laryngeal cancer remains unclear. Our study found that GAS5 and miR-21 has negative expression pattern in LSCC tissue. Meanwhile, GAS5 can negatively regulate miR-21 expression in laryngeal cell lines. miR-21 plays important roles in cancer progression, such as 
A

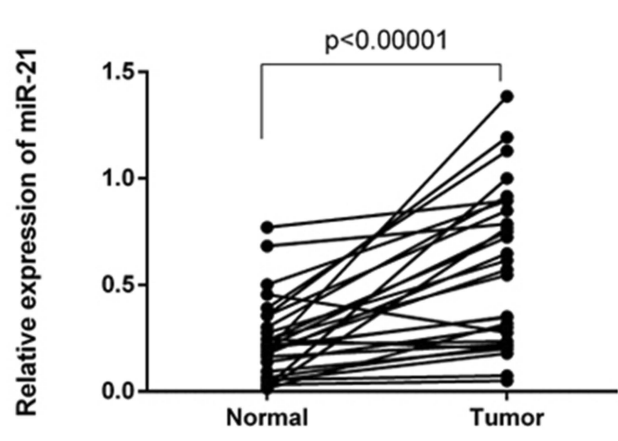

C

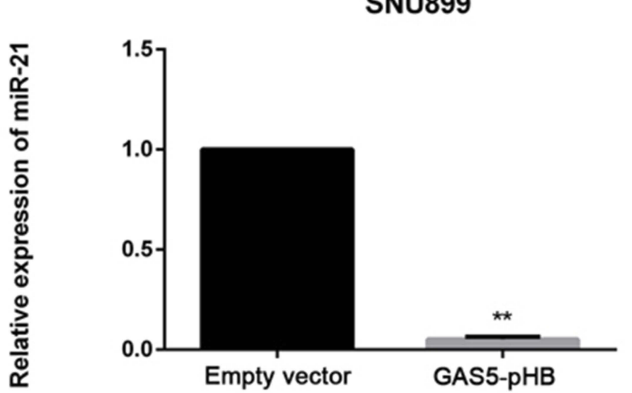

B

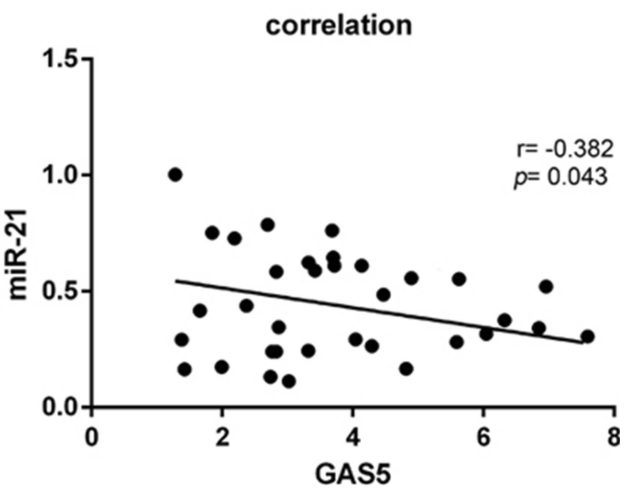

SNU1076

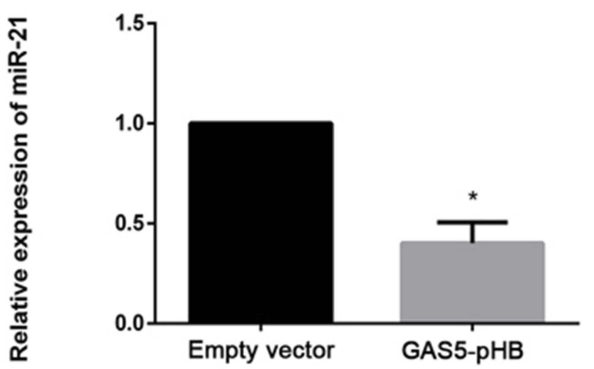

Figure 8 miR-2I is negatively regulated by GAS5. The relative expression of miR-2I in LSCC tissues ( $n=32$ ) and adjacent normal tissues were measured by RT-qPCR normalized to $\mathrm{U} 6$ expression (A). GAS5 and miR-2I expression in LSCC tissues $(n=32)$ measured by RT-qPCR were subject to co-expression analysis. The $x$-axis refers to GAS5 expression and Y-axis refers to miR-2I expression (B). Relative miR-2I expression was determined by RT-qPCR after GAS5 transfection (C, D). upregulated GAS5 significantly downregulated miR-2I expression in both SNU899 and SNUI076 cell lines, ${ }^{*} \mathrm{p}<0.05,{ }^{* *} \mathrm{p}<0.01$.

adenocarcinoma, esophageal cancer and lung cancer. ${ }^{37-39}$ Ectopic expression of miR-21 regulated BAX and CDK6 expression in this study. In addition, miR-21 reversed GAS5-media proliferation suppression and apoptosis. Similarly, miR-21 was confirmed to attenuate the regulation of BAX and CDK6 by GAS5. Collectively, this indicated that GAS5 suppressed proliferation and induced apoptosis through miR-21. However, this study has some limitations. GAS5 is not a novel lncRNA, instead an important lncRNA reported in other cancer. Our study furtherly elucidated the anti-tumor role of GAS5 in LSCC. Due to only functional and regulatory interaction evidence shown in this study, the binding sites of GAS5 and miR-21 still need further investigation to the clarify the specific mechanism of GAS5 in LSCC. Continued study of the specific mechanism and improved elucidation of GAS5 in LSCC hope to improve more effective and novel therapy strategies against LSCC.

In summary, the IncRNA GAS5 is downregulated in LSCC tissue compared with adjacent nontumor tissue. Lower expression of GAS5 is associated with T stage.
Then, ectopic expression of GAS5 suppressed cell proliferation and enhanced cell apoptosis in vivo. In addition, the overexpression of GAS5 increased BAX and decreased CDK6. Moreover, upregulation of GAS5 decreased the miR-21 level in the cell lines. The overexpression of miR-21 diminished the suppressed proliferative ability and enhanced the apoptotic effect induced by GAS5. As expected, upregulation of miR-21 reversed BAX and CDK6 expression by GAS5. The results showed that GAS5 regulates proliferation and apoptosis of LSCC cell lines through the regulation of miR-21 and its targets. This finding indicated that GAS5 may play an important role in LSCC progression and may be a novel and potential target for LSCC diagnosis and molecular therapy.

\section{Conclusion}

Down-regulated of IncRNA GAS5 contribute to the development of laryngeal cancer. GAS5 was found to be an antitumor gene that it can suppress cell proliferation and induce cell apoptosis by sponging to miR-21, thus 


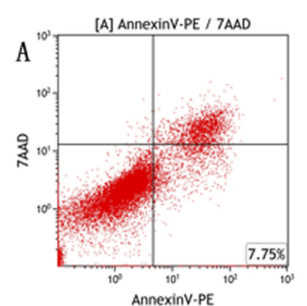

SNU899-Empty vector

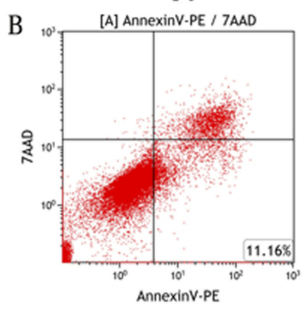

SNU899-GAS5-pHB
C

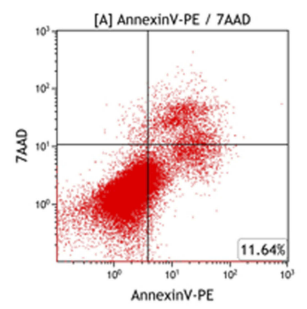

SNU899-GAS5-pHB+miR21-mimic-control

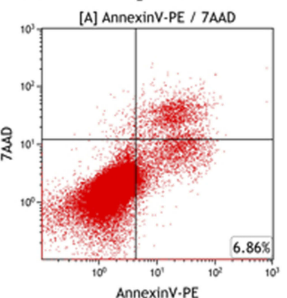

SNU899-GAS5-pHB+miR21-mimic
E

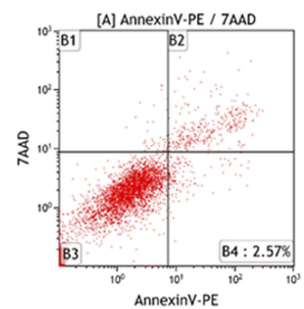

SNU1076-Empty vector

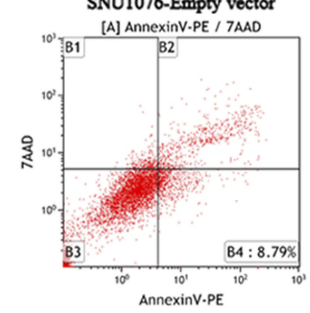

SNU1076-GAS5-pHB
G

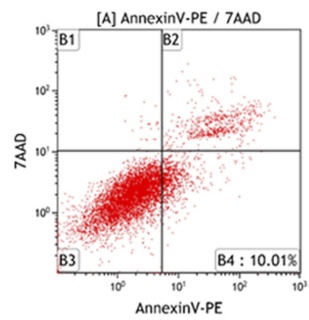

$\mathrm{H}$

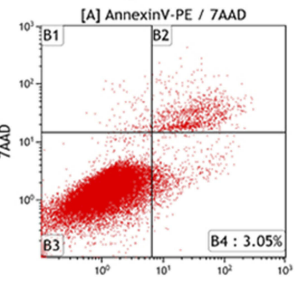

SNU1076-GAS5-pHB+miR21-mimic

I
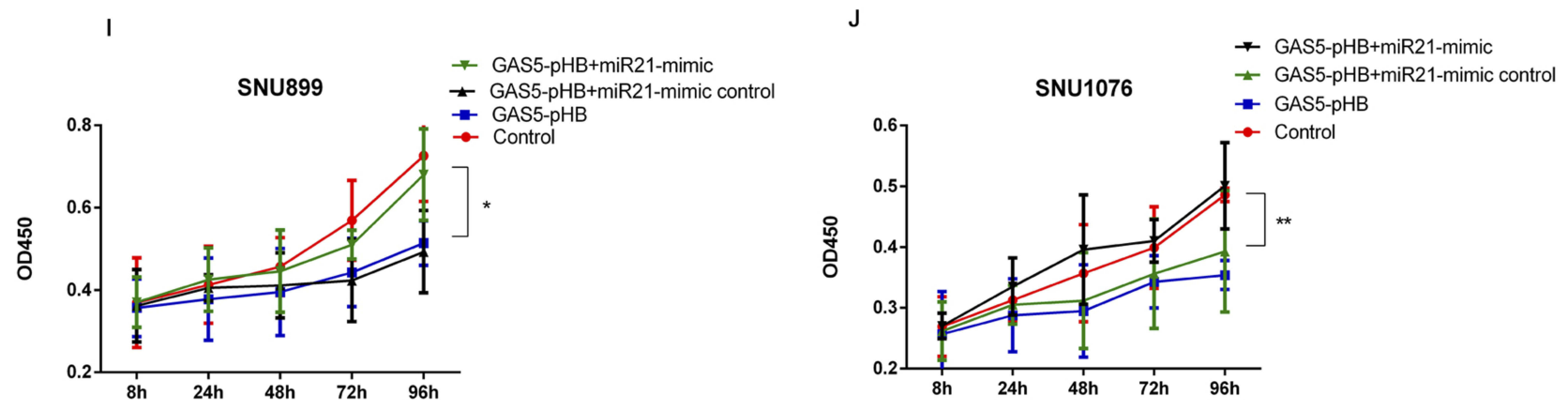

Figure 9 GAS5 suppressed LSCC progression via regulating miR-2I. SNU899 and SNU1076 cell lines were transfected with empty vector, GAS5-pHB, GAS5-pHB +miR-2I mimic control and GAS5-pHB+ miR-2I mimic. Cell apoptosis and proliferation were determined by flow cytometry and CCK8 assay, respectively. GAS5 mediated cell late apoptosis were eliminated by miR-2I in both SNU899 (A-D) and SNUI076 cell lines (E-H). (I-J) GAS5 mediated proliferation suppression was eliminated when GAS5 cotransfection with miR-2I mimic in both SNU899 and SNUI076 cell lines. ${ }^{*} p<0.05,{ }^{*} p<0.01$.

regulating its downstream genes BAX and CDK6. GAS5 may serve as a novel and potential target for LSCC diagnosis and molecular therapy.

\section{Acknowledgments}

This study was supported by the Science and Technology Program of Guangzhou of China (201704020092) and Science and Technology Planning Project of Guangdong Province of China (2017A050506018). Kexing Lyu , Yang $\mathrm{Xu}$ and Yun Li are co-first authors for this study.

\section{Data Sharing Statement}

The datasets used and/or analyzed during the current study are available from the corresponding author on reasonable request.

\section{Disclosure}

The authors report no conflicts of interest in this work.

\section{References}

1. Siegel RL, Miller KD, Jemal A. Cancer statistics, 2016. CA Cancer J Clin. 2016;66(1):7-30. doi:10.3322/caac.21332

2. Luo J, Wu J, Lv K, et al. Analysis of postsurgical health-related quality of life and quality of voice of patients with laryngeal carcinoma. Medicine. 2016;95(1):e2363. doi:10.1097/MD.0000000000004864

3. Baselga J. Why the epidermal growth factor receptor? The rationale for cancer therapy. Oncologist. 2002;7(Suppl 4):2-8. doi:10.1634/ theoncologist.7-suppl_4-2

4. Groome PA, O'Sullivan B, Irish JC, et al. Management and outcome differences in supraglottic cancer between Ontario, Canada, and the surveillance, epidemiology, and end results areas of the United States. J Clin Oncol. 2003;21(3):496-505. doi:10.1200/JCO. 2003.10.106

5. Kumar MS, Armenteros-Monterroso E, East P, et al. HMGA2 functions as a competing endogenous RNA to promote lung cancer progression. Nature. 2014;505(7482):212-217. doi:10.1038/nature12785

6. Tan DSW, Chong FT, Leong HS, et al. Long noncoding RNA EGFRAS1 mediates epidermal growth factor receptor addiction and modulates treatment response in squamous cell carcinoma. Nat Med. 2017;23(10):1167-1175. doi:10.1038/nm.4401

7. Song YX, Sun JX, Zhao JH, et al. Non-coding RNAs participate in the regulatory network of CLDN4 via ceRNA mediated miRNA evasion. Nat Commun. 2017;8(1):289. doi:10.1038/s41467-017-00304-1 
8. Faghihi MA, Modarresi F, Khalil AM, et al. Expression of a noncoding RNA is elevated in Alzheimer's disease and drives rapid feedforward regulation of beta-secretase. Nat Med. 2008;14(7):723-730. doi:10.1038/nm1784

9. Shen Z, Li Q, Deng H, Lu D, Song H, Guo J. Long non-coding RNA profiling in laryngeal squamous cell carcinoma and its clinical significance: potential biomarkers for LSCC. PLoS One. 2014;9(9): e108237. doi:10.1371/journal.pone.0108237

10. Li W, Sun M, Zang C, et al. Upregulated long non-coding RNA AGAP2-AS1 represses LATS2 and KLF2 expression through interacting with EZH2 and LSD1 in non-small-cell lung cancer cells. Cell Death Dis. 2016;7:e2225. doi:10.1038/cddis.2016.126

11. Schneider C, King RM, Philipson L. Genes specifically expressed at growth arrest of mammalian cells. Cell. 1988;54(6):787-793. doi:10. 1016/s0092-8674(88)91065-3

12. Kino T, Hurt DE, Ichijo T, Nader N, Chrousos GP. Noncoding RNA gas5 is a growth arrest- and starvation-associated repressor of the glucocorticoid receptor. Sci Signal. 2010;3(107):ra8. doi:10.1126/ scisignal.2000568

13. Mourtada-Maarabouni M, Pickard MR, Hedge VL, Farzaneh F, Williams GT. GAS5, a non-protein-coding RNA, controls apoptosis and is downregulated in breast cancer. Oncogene. 2009;28(2):195208. doi:10.1038/onc.2008.373

14. Sun M, Jin FY, Xia R, et al. Decreased expression of long noncoding RNA GAS5 indicates a poor prognosis and promotes cell proliferation in gastric cancer. BMC Cancer. 2014;14:319. doi:10.1186/14712407-14-319

15. Qiao H-P, Gao W-S, Huo J-X, Yang Z-S. Long non-coding RNA GAS5 functions as a tumor suppressor in renal cell carcinoma. Asian Pac $J$ Cancer Prev. 2013;14(2):1077-1082. doi:10.7314/apjcp.2013.14.2.1077

16. Ren J, Zhu D, Liu M, Sun Y, Tian L. Downregulation of miR-21 modulates Ras expression to promote apoptosis and suppress invasion of Laryngeal squamous cell carcinoma. Eur J Cancer. 2010;46 (18):3409-3416. doi:10.1016/j.ejca.2010.07.047

17. $\mathrm{Hu} \mathrm{A}$, Huang JJ, Xu WH, et al. miR-21 and miR-375 microRNAs as candidate diagnostic biomarkers in squamous cell carcinoma of the larynx: association with patient survival. Am J Transl Res. 2014;6 (5):604-613.

18. Huarte M. The emerging role of 1 ncRNAs in cancer. Nat Med. 2015;21(11):1253-1261. doi:10.1038/nm.3981

19. Lee JT. Epigenetic regulation by long noncoding RNAs. Science. 2012;338(6113):1435-1439. doi:10.1126/science.1231776

20. Salmena L, Poliseno L, Tay Y, Kats L, Pandolfi PP. A ceRNA hypothesis: the Rosetta Stone of a hidden RNA language? Cell. 2011;146(3):353-358. doi:10.1016/j.cell.2011.07.014

21. Zhang Z, Zhu Z, Watabe $K$, et al. Negative regulation of lncRNA GAS5 by miR-21. Cell Death Differ. 2013;20(11):1558-1568. doi: $10.1038 /$ cdd.2013.110

22. Steuer CE, El-Deiry M, Parks JR, Higgins KA, Saba NF. An update on larynx cancer. CA Cancer J Clin. 2017;67(1):31-50. doi:10.3322/ caac. 21386

23. Tang JY, Lee JC, Chang YT, et al. Long noncoding RNAs-related diseases, cancers, and drugs. TheScientificWorldJournal. 2013; 2013:943539. doi:10.1155/2013/943539

24. Pope C, Mishra S, Russell J, Zhou Q, Zhong XB. Targeting H19, an imprinted long non-coding RNA, in hepatic functions and liver diseases. Diseases. 2017;5:1. doi:10.3390/diseases5010011
25. Song H, Sun W, Ye G, et al. Long non-coding RNA expression profile in human gastric cancer and its clinical significances. $J$ Transl Med. 2013;11:225. doi:10.1186/1479-5876-11-225

26. Zhao J, Lv K, Li ZH, et al. Functional significance of the long noncoding RNA RP11-169D4.1 as a metastasis suppressor in laryngeal squamous cell carcinoma by regulating CDH1. Oncol Rep. 2017;38 (1):211-220. doi:10.3892/or.2017.5645

27. Chang L, Li C, Lan T, et al. Decreased expression of long noncoding RNA GAS5 indicates a poor prognosis and promotes cell proliferation and invasion in hepatocellular carcinoma by regulating vimentin. Mol Med Rep. 2016;13(2):1541-1550. doi:10.3892/ mmr.2015.4716

28. Krell J, Frampton AE, Mirnezami R, et al. Growth arrest-specific transcript 5 associated snoRNA levels are related to p53 expression and DNA damage in colorectal cancer. PLoS One. 2014;9(6):e98561. doi:10.1371/journal.pone.0098561

29. Shi X, Sun M, Liu H, et al. A critical role for the long non-coding RNA GAS5 in proliferation and apoptosis in non-small-cell lung cancer. Mol Carcinog. 2015;54(Suppl 1):E1-E12. doi:10.1002/ mc. 22120

30. Pickard MR, Williams GT. Regulation of apoptosis by long noncoding RNA GAS5 in breast cancer cells: implications for chemotherapy. Breast Cancer Res Treat. 2014;145(2):359-370. doi:10. 1007/s10549-014-2974-y

31. Pickard MR, Mourtada-Maarabouni M, Williams GT. Long noncoding RNA GAS5 regulates apoptosis in prostate cancer cell lines Biochim Biophys Acta. 2013;1832(10):1613-1623. doi:10.1016/j. bbadis.2013.05.005

32. Guo X, Deng K, Wang H, et al. GAS5 inhibits gastric cancer cell proliferation partly by modulating CDK6. Oncol Res Treat. 2015;38 (7-8):362-366. doi:10.1159/000433499

33. Bosson AD, Zamudio JR, Sharp PA. Endogenous miRNA and target concentrations determine susceptibility to potential ceRNA competition. Mol Cell. 2014;56(3):347-359. doi:10.1016/j.molcel.2014.09. 018

34. Wang Y, Sun L, Wang L, et al. Long non-coding RNA DSCR8 acts as a molecular sponge for miR-485-5p to activate Wnt/beta-catenin signal pathway in hepatocellular carcinoma. Cell Death Dis. 2018;9 (9):851. doi:10.1038/s41419-018-0937-7

35. Yong W, Yu D, Jun Z, et al. Long noncoding RNA NEAT1, regulated by LIN28B, promotes cell proliferation and migration through sponging miR-506 in high-grade serous ovarian cancer. Cell Death Dis. 2018;9(9):861. doi:10.1038/s41419-018-0908-z

36. Song J, Ahn C, Chun CH, Jin EJ. A long non-coding RNA, GAS5, plays a critical role in the regulation of miR-21 during osteoarthritis. J Orthop Res. 2014;32(12):1628-1635. doi:10.1002/jor.22 718

37. Zheng W, Zhao J, Tao Y, et al. MicroRNA-21: A promising biomarker for the prognosis and diagnosis of non-small cell lung cancer. Oncol Lett. 2018;16(3):2777-2782. doi:10.3892/ol.2018.8972

38. Xia Y, Wang Y, Wang Q, et al. Increased miR-203-3p and reduced miR-21-5p synergistically inhibit proliferation, migration, and invasion in esophageal cancer cells. Anti-cancer Drugs. 2019;30(1):3845. doi:10.1097/CAD.0000000000000687

39. Yu W, Zhu K, Wang Y, Yu H, Guo J. Overexpression of miR-21-5p promotes proliferation and invasion of colon adenocarcinoma cells through targeting CHL1. Mol Med. 2018;24(1):36. 


\section{Publish your work in this journal}

Cancer Management and Research is an international, peer-reviewed open access journal focusing on cancer research and the optimal use of preventative and integrated treatment interventions to achieve improved outcomes, enhanced survival and quality of life for the cancer patient.
The manuscript management system is completely online and includes a very quick and fair peer-review system, which is all easy to use. Visit http://www.dovepress.com/testimonials.php to read real quotes from published authors. 